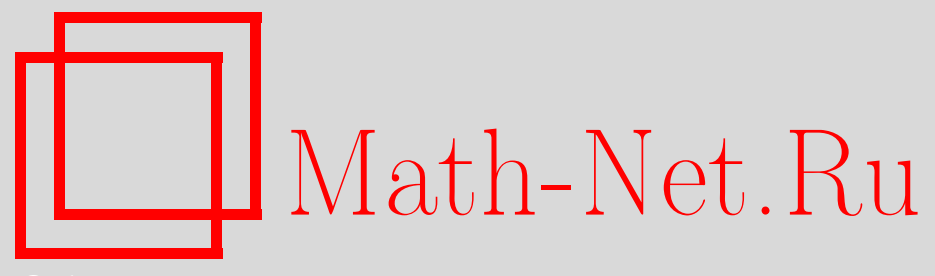

Г. Н. Белосточный, О. А. Мыльцина, Динамическая термоустойчивость геометрически нерегулярной пологой цилиндрической оболочки под действием периодической, по временной координате, нагрузки, Вестн. Сам. гос. техн. ун-та. Сер. Физ.-мат. науки, 2020, номер 3, 583-594

DOI: https://doi.org/10.14498/vsgtu1755

Использование Общероссийского математического портала MathNet.Ru подразумевает, что вы прочитали и согласны с пользовательским соглашением

http://www.mathnet.ru/rus/agreement

Параметры загрузки:

IP: 107.22 .136 .117

26 апреля 2023 г., 12:31:17

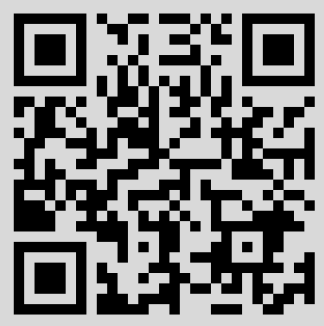


УДК 517.958:539.3(1)

\title{
Динамическая термоустойчивость геометрически нерегулярной пологой цилиндрической оболочки под действием периодической по временной координате нагрузки
}

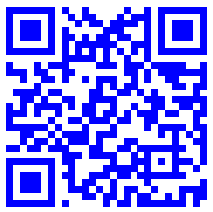

\section{(С) Г. Н. Белосточный, О. А. Мылъиина}

Саратовский государственный университет им. Н. Г. Чернышевского (национальный исследовательский университет),

Россия, 410012, Саратов, ул. Астраханская, 83.

\begin{abstract}
Аннотация
В рамках модели типа Лява рассматривается геометрически нерегулярная изотропная пологая цилиндрическая оболочка (ГНО). За основу берется строгая континуальная модель «оболочка-ребра». Предполагается, что ГНО нагрета до постоянной температуры $\theta_{0}$, два противоположных края подвергаются воздействию периодической по временной координате тангенциальной нагрузке, амплитуда и частота которой известны $\left(p(t)=p_{0} \cos \vartheta t\right)$. Задача определения динамической неустойчивости (ДН) термоупругой системы сводится к рассмотрению сингулярной системы трех дифференциальных уравнений динамической термоустойчивости ГНО в перемещениях, содержащих слагаемые с тангенциальными усилиями в форме Брайена. Эти усилия, возникающие в оболочке при ее нагреве, предварительно определяются на основе замкнутых решений сингулярной системы дифференциальных уравнений безмоментной термоупругости ГНО. Конкретизированная исходная система уравнений преобразуется к уравнениям Матье, которые записаны в терминах классической атермической теории гладких пластин, содержащих поправки на геометрические параметры - кривизну, относительную высоту подкрепляющих элементов, их число и температуру. Определяются первые три области ДН ГНО. Проводится количественный анализ влияния геометрических параметров упругой системы и температуры на конфигурацию областей ДН и предельного значения коэффициента возбуждения.
\end{abstract}

\section{Краткое сообщение}

(웅 Контент публикуется на условиях лицензии Creative Commons Attribution 4.0 International (https://creativecommons.org/licenses/by/4.0/deed.ru)

\section{Образец для цитирования}

Бел осточны й Г. Н., М ыльцина О. А. Динамическая термоустойчивость геометрически нерегулярной пологой цилиндрической оболочки под действием периодической по временной координате нагрузки // Вестн. Сам. гос. техн. ун-та. Сер. Физ.-мат. науки, 2020. T. 24, № 3. C. 583-594. https://doi.org/10.14498/vsgtu1755.

\section{Сведения об авторах}

Григорий Николаевич Белосточный (1) https://orcid.org/0000-0003-4471-6599 доктор технических наук, профессор; профессор; каф. математической теории упругости и биомеханики; e-mail: belostochny@mail.ru

Ольга Анатольевна Мыльцина (10) https://orcid.org/0000-0003-4718-2772

кандидат физико-математических наук; доцент; каф. теории функций и стохастического анализа; e-mail: omyltcina@yandex.ru 
Ключевые слова: сингулярность, термоустойчивость, динамика, геометрическая нерегулярность, континуальная модель, уравнения Матье, замкнутые интегралы, области неустойчивости.

Получение: 14 ноября 2019 г. / Исправление: 25 июня 2020 г. Принятие: 14 сентября 2020 г. / Публикация онлайн: 28 сентября 2020 г.

На базе континуальной модели [1-4] рассмотрим геометрически нерегулярную пологую цилиндрическую оболочку, стандартным образом отнесенную к декартовым координатам [5], перекрывающую в координатной плоскости $\pi\left(x_{1} O x_{2}\right)$ прямоугольный план со сторонами $a$ и $b$ соответственно. Оболочка нагрета до постоянной температуры $\theta_{0}$, на торцах оболочки, расположенных по координатным прямым $x_{2}=0$ и $x_{2}=b$, действуют периодические по временной координате тангенциальные нагрузки заданной интенсивности $p(t)=p_{0} \cos \vartheta t$.

Система сингулярных дифференциальных уравнений динамической термоустойчивости геометрически нерегулярной цилиндрической оболочки в компонентах поля перемещений запишется в виде [3]

$$
\begin{aligned}
& u, 11+\frac{1-\nu}{2} u,,_{22}+\frac{1+\nu}{2} v,_{12}+k_{11} w,_{1}+ \\
& +\varepsilon_{1} \sum_{i=1}^{n} \frac{h_{i}}{h} a_{i}\left(u,,_{2}+v,{ }_{1}\right),{ }_{2} \delta\left(x-x_{i}\right)=0, \\
& \frac{1+\nu}{2} u,,_{12}+v,_{22}+\frac{1-\nu}{2} v,,_{11}-\nu k_{11} w,_{2}+ \\
& +\varepsilon_{2} \sum_{i=1}^{n} \frac{h_{i}}{h} a_{i}\left(v, 2+\nu\left(u,{ }_{1}-k_{11} w\right)\right){ }_{2} \delta\left(x-x_{i}\right)=0, \\
& \nabla^{2} \nabla^{2} w+\frac{B}{D} k_{11}^{2} w-\frac{B}{D}\left(u,{ }_{1}+\nu v,,_{2}\right) k_{11}+\frac{T_{0}^{11}}{D} w,,_{11}+2 \frac{T_{0}^{12}}{D} w,,_{12}+\frac{T_{0}^{22}}{D} w, 22_{22}+ \\
& +\sum_{i=1}^{n}\left(\frac{h_{i}}{h}\right)^{3} a_{i} \Phi_{3 i} w, 2222 \delta\left(x-x_{i}\right)+2(1-\nu) \sum_{i=1}^{n}\left(\frac{h_{i}}{h}\right)^{3} a_{i} \Phi_{3 i} w,{ }_{122} \mid \frac{d \delta\left(x-x_{i}\right)}{x_{i}}= \\
& =-\frac{\gamma h}{g D} w,{ }_{t t}-\sum_{i=1}^{n} \frac{\gamma h_{i}}{g D} a_{i} w,{ }_{t t} \delta\left(x-x_{i}\right) .
\end{aligned}
$$

Здесь $\varepsilon_{j}(j=1,2)$ - знаковые числа, равные 0 или $1 ; \nu$ - коэффициент Пуассона; $k_{11}$ - параметр кривизны; $\bar{U}(u, v, w)$ - компоненты поля перемещений, когда оболочки находится в моментном состоянии; $B=\frac{E h}{1-\nu^{2}} ; D=\frac{E h^{3}}{12\left(1-\nu^{2}\right)}$; $h_{i} / h$ - отношение высоты $i$-того ребра к толщине оболочки; $a_{i}$ - ширина $i$-того ребра; $\Phi_{3 i}=1+3 \frac{h}{h_{i}}+3\left(\frac{h}{h_{i}}\right)^{2} ; E$ - модуль Юнга; $\gamma$ - удельный вес; $g$ - интенсивность поля тяжести; $\delta\left(x-x_{i}\right)$ - обобщенная $\delta$-функция Дирака [6].

Подчеркнутые слагаемые в первых двух уравнениях (1) соответствуют учету «растяжения-сжатия» и «сдвига» ребер в тангенциальной плоскости в терминах дискретной модели [7]. 
$T_{0}^{11}, T_{0}^{12}, T_{0}^{22}$ - тангенциальные усилия, возникающие в ГНО при нагреве и силовом воздействии на торцы в ее безмоментном состоянии, содержатся в форме Брайена [8]. Отметим, что в первых двух уравнениях системы (1) отсутствуют инерционные члены в тангенциальной плоскости оболочки $[9,10]$.

В случае краевых условий

$$
\begin{array}{ll}
\text { при } x_{1}=0, x_{1}=a & u^{0}=0, T_{0}^{12}=0, \\
\text { при } x_{2}=0, x_{2}=b & T_{0}^{22}=-p(t), T_{0}^{12}=0,
\end{array}
$$

которые в перемещениях перепишутся в виде

$$
\begin{array}{cl}
\text { при } x_{1}=0, x_{1}=a & u^{0}=0, u_{, 2}^{0}+v_{, 1}^{0}=0, \\
\text { при } x_{2}=0, x_{2}=b & v_{, 2}^{0}+\nu\left(u_{, 1}^{0}-k_{11} w^{0}\right)=-\frac{p(t)}{B}+\alpha(1+\nu) \theta_{0}, \\
& u_{, 2}^{0}+v_{, 1}^{0}=0,
\end{array}
$$

решение безмоментной термоупругости ГНО запишется так [11-14]:

$$
u^{0}=w^{0}=0, v^{0}=\alpha(1+\nu) \theta_{0} x_{2}-2 \frac{p(t)}{B} x_{2},
$$

и, следовательно, тангенциальные усилия в третьем уравнении системы (1) примут вид

$$
T_{0}^{11}=-(1-\nu) B \alpha \theta_{0}-\nu p(t), \quad T_{0}^{22}=-p(t), \quad T_{0}^{12}=0 .
$$

Здесь $\bar{U}^{0}\left(u^{0}, v^{0}, w^{0}\right)$ - компоненты поля перемещений в безмоментном состоянии ГНО, $\alpha$ - коэффициент линейного расширения материала.

Решения системы (1) с учетом вида тангенциальных усилий (2), тождественно удовлетворяющих всем краевым условиям (для случая, когда ГНО находится в моментном состоянии)

$$
\begin{aligned}
& \text { при } x_{1}=0, x_{1}=a \quad u=0, T^{12}=0, w=0, M^{11}=0 \\
& \quad(u=0, u, 2+v, 1=0, w=0, w, 11=0) ; \\
& \text { при } x_{2}=0, x_{2}=b \quad T^{22}=0, T^{12}=0, w=0, M^{22}=0 \\
& \quad\left(v, 2+\nu\left(u, 1-k_{11} w\right)=0, u, 2+v, 1=0, w=0, w, 22=0\right)
\end{aligned}
$$

зададим в виде

$$
\begin{aligned}
u\left(x_{1}, x_{2}, t\right) & =\widetilde{u}(t)\left(\frac{x_{1}}{a}\right)^{2}\left(\frac{x_{1}}{a}-1\right)\left(\frac{x_{2}}{b}\right)^{3}\left(\frac{x_{2}}{b}-1\right)^{2}, \\
v\left(x_{1}, x_{2}, t\right) & =\widetilde{v}(t)\left(\frac{x_{1}}{a}\right)^{3}\left(\frac{x_{1}}{a}-1\right)^{2}\left(\frac{x_{2}}{b}\right)^{3}\left(\frac{x_{2}}{b}-1\right)^{2}, \\
w\left(x_{1}, x_{2}, t\right) & =w_{k m}(t) \sin \frac{k \pi x_{1}}{a} \sin \frac{m \pi y}{b} .
\end{aligned}
$$

В условиях (3) в скобках приводится их запись в компонентах поля перемещений. 
Подстановка (4) в уравнения системы (1) с последующим применением процедуры Галеркина приводит к трем уравнениям относительно переменных коэффициентов аппроксимирующих функций (4):

$$
\begin{aligned}
& e_{11} \widetilde{u}+e_{12} \widetilde{v}=-4 \frac{\widetilde{\delta}}{h} \frac{h}{a} \frac{a}{b} I^{4} w_{k m}, \quad e_{21} \widetilde{u}+e_{22} \widetilde{v}=-4 \nu \frac{\widetilde{\delta}}{h} \frac{h}{a} \frac{a}{b} I^{8} w_{k m} \\
& \frac{\gamma h a^{4}}{g D}\left(1+2 \sum_{i=1}^{n} \widetilde{\beta}_{i}^{s}\right) \frac{d^{2} w_{k m}}{d t^{2}}+\left((k \pi)^{2}+\left(\frac{m \pi a}{b}\right)^{2}\right)^{2}+192\left(\frac{\widetilde{\delta}}{h}\right)^{2}+ \\
& +48\left(\frac{a}{h}\right)^{2} \frac{\widetilde{\delta}}{h} \frac{h}{a} \frac{a}{b}\left(\widetilde{u} \frac{b}{a} I^{9}+\nu I^{10} \widetilde{v}\right)-12\left(1-\nu^{2}\right)(k \pi)^{2}\left(\frac{a}{h}\right)^{2}- \\
& -12 \frac{p(t)}{E h}\left(1-v^{2}\right)\left(\frac{a}{h}\right)^{2}\left(\left(\frac{m \pi}{b}\right)^{2}+\nu(k \pi)^{2}+2\left(\frac{m \pi a}{b}\right)^{2} \sum_{i=1}^{n} \widetilde{\beta}_{i}^{s}+\right. \\
& \left.+2\left(\frac{m \pi a}{b}\right)^{4} \sum_{i=1}^{n} \beta_{i}^{s}+4(1-\nu)\left(\frac{m \pi a}{b}\right)^{2}(k \pi)^{2} \sum_{i=1}^{n} \beta_{i}^{c}\right) w_{k m}(t)=0 .
\end{aligned}
$$

Здесь $\widetilde{\delta} / h$ - относительная стрела подъема оболочки над ее планом при задании относительного параметра кривизны в виде $k_{11} a=-4 \frac{\widetilde{\delta}}{h} \frac{h}{a}[5,7]$;

$$
\begin{gathered}
e_{11}=\frac{b}{a} I^{1}+\frac{1-\nu}{2} \frac{a}{b} I^{2}+\frac{1-\nu}{2} \varepsilon_{1} \frac{a}{b} \sum_{i=1}^{n} \frac{h_{i}}{h} \frac{a_{i}}{a} G^{1 i}, \\
e_{12}=\frac{1+\nu}{2} I^{3}+\frac{1-\nu}{2} \varepsilon_{1} \sum_{i=1}^{n} \frac{h_{i}}{h} \frac{a_{i}}{a} G^{2 i}, \quad e_{21}=\frac{1+\nu}{2} I^{5}+\nu \varepsilon_{2} \sum_{i=1}^{n} \frac{h_{i}}{h} \frac{a_{i}}{a} G^{3 i}, \\
e_{22}=\frac{a}{b} I^{6}+\frac{1-\nu}{2} \frac{b}{a} I^{7}+\varepsilon_{2} \frac{a}{b} \sum_{i=1}^{n} \frac{h_{i}}{h} \frac{a_{i}}{a} G^{4 i} ; \\
I^{3}=\int_{0}^{1} \int_{0}^{1} \int_{0}^{1}(6 X-2)\left(X^{3}-8 X^{3}+3 X^{2}\right)\left(X^{3}-X^{5}\right)\left(5 Y^{4}-8 Y^{3}+3 Y^{2}\right) \times \\
I^{7}=\int_{0}^{1} \int_{0}^{1}\left(20 X^{3}-24 X^{2}+6 X\right)\left(X^{5}-2 X^{4}+X^{3}\right)\left(Y^{5}-2 Y^{4}+Y^{3}\right)^{2} d X d Y, \ldots\left(Y^{5}-2 Y^{4}+3 Y^{3}\right) d X d Y, \\
I^{6}=\int_{0}^{1} \int_{0}^{1}\left(X^{5}-2 X^{4}+X^{3}\right)^{2}\left(20 Y^{3}-24 Y^{2}+6 Y\right)\left(Y^{5}-2 Y^{4}+Y^{3}\right) d X d Y, \\
\times \sin y\left(\left(\frac{x}{m \pi}\right)^{3}-\left(\frac{x}{k \pi}\right)^{2}\right) \times \\
\left.\int^{5}-2\left(\frac{y}{m \pi}\right)^{4}+\left(\frac{y^{3}}{m \pi}\right)^{3}\right) d x d y,
\end{gathered}
$$




$$
\begin{gathered}
I^{10}=\frac{1}{k \pi} \frac{1}{m \pi} \int_{0}^{k \pi} \int_{0}^{m \pi} \sin x\left(\left(\frac{x}{k \pi}\right)^{5}-2\left(\frac{x}{k \pi}\right)^{4}+\left(\frac{x}{k \pi}\right)^{3}\right) \times \\
\times \sin y\left(5\left(\frac{y}{m \pi}\right)^{4}-8\left(\frac{y}{m \pi}\right)^{3}+3\left(\frac{y}{m \pi}\right)^{2}\right) d x d y \\
G^{1 i}=\left(\left(\frac{x_{i}}{a}\right)^{3}-\left(\frac{x_{i}}{a}\right)^{2}\right)^{2} \int_{0}^{1}\left(20 Y^{3}-24 Y^{2}+6 Y\right)\left(Y^{5}-2 Y^{4}+Y^{3}\right) d Y, \ldots, \\
G^{4 i}=\left(\left(\frac{x_{i}}{a}\right)^{5}-2\left(\frac{x_{i}}{a}\right)^{4}+\left(\frac{x_{i}}{a}\right)^{3}\right)^{2} \int_{0}^{1}\left(20 Y^{3}-24 Y^{2}+6 Y\right)\left(Y^{5}-2 Y^{4}+Y^{3}\right) d Y \\
\beta_{i}^{s}=\left(\frac{h_{i}}{h}\right)^{3} \frac{a_{i}}{a} \Phi_{3 i} \sin ^{2} \frac{k \pi x_{i}}{a}, \beta_{i}^{c}=\left(\frac{h_{i}}{h}\right)^{3} \frac{a_{i}}{a} \Phi_{3 i} \cos ^{2} \frac{k \pi x_{i}}{a}, \quad \widetilde{\beta}_{i}^{s}=\frac{h_{i}}{h} \frac{a_{i}}{a} \sin ^{2} \frac{k \pi x_{i}}{a} .
\end{gathered}
$$

Выражая из первых двух уравнений системы $(5) \widetilde{u}$ и $\widetilde{v}$ через $w_{k m}$, перепишем третье уравнение после ряда преобразований в виде

$$
\begin{aligned}
& \frac{d^{2} w_{k m}}{d t^{2}}+\frac{\left((k \pi)^{2}+\left(\frac{m \pi a}{b}\right)^{2}\right)^{2}}{\frac{\gamma h a^{4}}{g D}} \frac{f_{2}^{k m}}{f_{3}^{k m}} \times \\
& \quad \times\left(1-\frac{12\left(1-\nu^{2}\right)\left(\frac{a}{h}\right)^{2}\left(\nu(k \pi)^{2}+\left(\frac{m \pi a}{b}\right)^{2}\right)^{2}}{\left((k \pi)^{2}+\left(\frac{m \pi a}{b}\right)^{2}\right)^{2}} \frac{f_{1}^{k m}}{f_{2}^{k m}} \frac{p_{0}}{E h} \cos \vartheta t\right) w_{k m}=0 .
\end{aligned}
$$

Это есть уравнение Матье [15, 16], записанное в терминах классической атермической теории пластин с поправками на параметры $\frac{\widetilde{\delta}}{h}, \frac{h_{i}}{h}, n$ и $\theta_{0}$. Здесь

$$
\begin{gathered}
f_{1}^{k m}=1+\frac{2\left(\frac{m \pi a}{b}\right)^{2} \sum_{i=1}^{n} \widetilde{\beta}_{i}^{s}}{\left(\frac{m \pi a}{b}\right)^{2}+\nu(k \pi)^{2}}, \quad f_{3}^{k m}=1+2 \sum_{i=1}^{n} \widetilde{\beta}_{i}^{s}, \\
f_{2}^{k m}=1+\frac{192 \Gamma_{k m}\left(\frac{\widetilde{\delta}}{h}\right)^{2}+\sum_{i=1}^{n} \beta_{i}-12\left(1-\nu^{2}\right)(k \pi)^{2}\left(\frac{a}{h}\right)^{2} \alpha \theta_{0}}{\left((k \pi)^{2}+\left(\frac{m \pi a}{b}\right)^{2}\right)^{2}}, \\
\beta_{i}=2\left(\frac{m \pi a}{b}\right)^{4} \beta_{i}^{s}+4\left(\frac{m \pi a}{b}\right)^{2}(k \pi)^{2}(1-\nu) \beta_{i}^{c}, \\
\Gamma_{k m}=1-\frac{a}{b}\left(\frac{b}{a} I^{9} \frac{\frac{a}{b} I^{4} e_{22}-\nu I^{8} e_{12}}{e_{11} e_{22}-e_{12} e_{21}}+\nu I^{10} \frac{\nu I^{8} e_{11}-\frac{a}{b} I^{4} e_{21}}{e_{11} e_{22}-e_{12} e_{21}}\right) .
\end{gathered}
$$

Следует отметить, что $\left((k \pi)^{2}+\left(\frac{m \pi a}{b}\right)^{2}\right)^{2} /\left(\frac{\gamma h a^{4}}{g D}\right)-$ квадрат частоты собственных колебаний гладкой пластинки [17, 18],

$$
\frac{12\left(1-\nu^{2}\right)\left(\frac{a}{b}\right)^{2}\left(\nu(k \pi)^{2}+\left(\frac{m \pi a}{b}\right)^{2}\right)^{2}}{\left((k \pi)^{2}+\left(\frac{m \pi a}{b}\right)^{2}\right)^{2}}=\frac{E h}{p_{0}^{\mathrm{Kp}}}
$$

- величина, обратная относительной критической нагрузке при статической потере устойчивости [19], $\mu_{k m}=0.5 p_{0} / p_{\text {кр }}$ - коэффициент возбуждения гладкой пластинки на базе атермической теории, предельное значение которого $\widetilde{\mu}_{k m}=0.5$ [20]. Границы первых трех областей неустойчивости [16] в системе $\left\{\mu_{k m}, \frac{\vartheta}{\omega_{k m}}\right\}$ примут вид 


$$
\begin{gathered}
\frac{\vartheta}{\omega_{k m}}=2 \sqrt{\frac{f_{2}^{k m}}{f_{3}^{k m}} \pm \frac{f_{1}^{k m}}{f_{3}^{k m}} \mu_{k m}} ; \\
\frac{\vartheta}{\omega_{k m}}=\sqrt{\frac{f_{2}^{k m}}{f_{3}^{k m}}-2 \frac{\left(f_{1}^{k m}\right)^{2}}{f_{2}^{k m} f_{3}^{k m}} \mu_{k m},} \\
\frac{\vartheta}{\omega_{k m}}=\sqrt{\frac{5}{8} \frac{f_{2}^{k m}}{f_{3}^{k m}}+\frac{1}{2} \frac{f_{2}^{k m}}{f_{3}^{k m}} \sqrt{\frac{9}{16}+\left(\frac{f_{1}^{k m}}{f_{2}^{k m}}\right)^{2} \mu_{k m}}}
\end{gathered}
$$

$$
\frac{\vartheta}{\omega_{k m}}=\sqrt{\frac{f_{2}^{k m}}{f_{3}^{k m}}\left(\frac{10}{9} \pm \frac{f_{1}^{k m}}{f_{2}^{k m}} \mu_{k m}-\sqrt{\left(\frac{10}{9} \pm \frac{f_{1}^{k m}}{f_{2}^{k m}} \mu_{k m}\right)^{2}-\frac{4}{9}\left(1 \pm \frac{f_{1}^{k m}}{f_{2}^{k m}} \mu_{k m}-\left(\frac{f_{1}^{k m}}{f_{2}^{k m}} \mu_{k m}\right)^{2}\right)}\right)}
$$

Приведенного уравнения (6) и формул (7) достаточно для определения влияния геометрических параметров на предельное значение коэффициента возбуждения и границы областей динамической неустойчивости при любых значениях $k$ и $m$. Ниже приведены предельные значения $\widetilde{\mu}_{k m}$ в зависимости от величины параметра $\widetilde{\delta} / h$ при прочих равных условиях.

\begin{tabular}{|c|c|c|}
\hline$\tilde{\delta} / h$ & $n=0$ & $n=3, h_{i} / h=5$ \\
\hline 1 & 0.33629 & 0.16245 \\
2 & 0.16965 & 0.11275 \\
3 & 0.09291 & 0.07468 \\
4 & 0.05688 & 0.05070 \\
5 & 0.03794 & 0.03589 \\
\hline
\end{tabular}

Здесь и далее $\varepsilon_{1}=\varepsilon_{2}=0, k=1, m=1, a / b=1$ (если не указано иного), $a_{i} / a=0.005$ (если есть ребра).

Количественные результаты, полученные на основании формул (7), (8)(10), приводятся на рисунках ниже.

Из представленных рисунков следует, что конфигурации областей динамической неустойчивости малочувствительны к величине температуры (в предельных значениях не превышают потерю статической термоустойчивости). Следует отметить важный факт, что при соответствующем выборе условий закрепления можно полностью избежать влияния температуры на области динамической неустойчивости. Действительно, принимая условия закрепления в виде

$$
\begin{aligned}
& \text { при } x_{1}=0, x_{1}=a \quad T_{0}^{12}=0, T_{0}^{11}=0 \\
& \quad\left(u_{, 2}^{0}+v_{, 1}^{0}=0, u_{,_{1}}^{0}+v_{, 2}^{0}-k_{11} w^{0}=\alpha(1+\nu) \theta_{0}\right) ; \\
& \text { при } x_{2}=0, x_{2}=b \quad T^{22}=-p(t), T^{12}=0, \\
& \quad\left(v, 2+\nu\left(u,,_{1}-k_{11} w\right)=\alpha(1+\nu) \theta_{0}-p(t) / B, u,,_{2}+v,_{1}=0\right)
\end{aligned}
$$

(здесь, как и выше, приводятся условия закрепления в перемещениях), решение безмоментной термоупругости ГНО можно записать в виде [11]

$$
u^{0}=\frac{\nu p(t)}{B\left(1-\nu^{2}\right)} x_{1}+\alpha \theta_{0} x_{1}, \quad v^{0}=-\frac{p(t)}{B\left(1-v^{2}\right)} x_{2}+\alpha \theta_{0} x_{2}, \quad w^{0}=0 .
$$




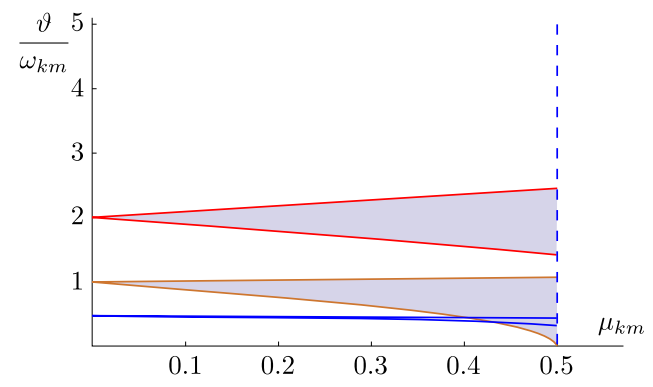

$\widetilde{\mu}_{k m}=0.5, n=0, \widetilde{\delta} / h=0, \theta_{0}=0$

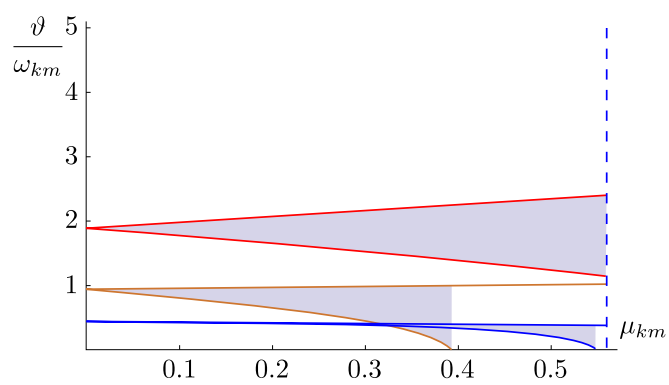

$\tilde{\mu}_{k m}=0.56, n=0, \widetilde{\delta} / h=0, \theta_{0}=20$

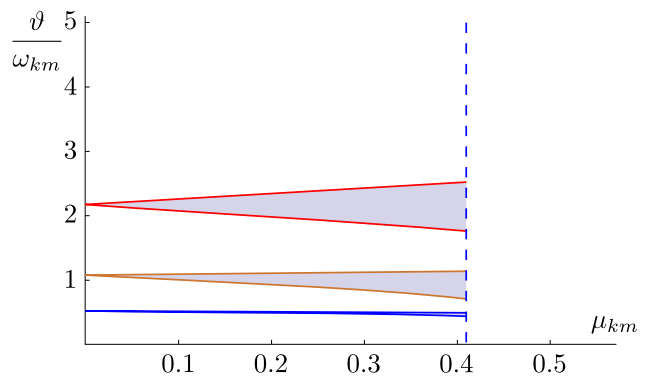

$\widetilde{\mu}_{k m}=0.41, n=3, h_{i} / h=2, \widetilde{\delta} / h=0, \theta_{0}=0$

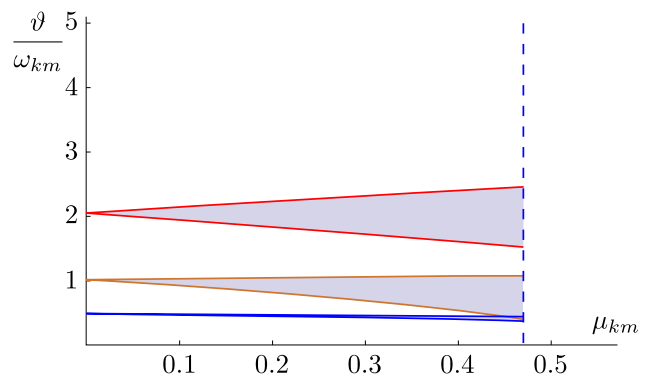

$\widetilde{\mu}_{k m}=0.47, n=1, h_{i} / h=2, \widetilde{\delta} / h=0, \theta_{0}=0$

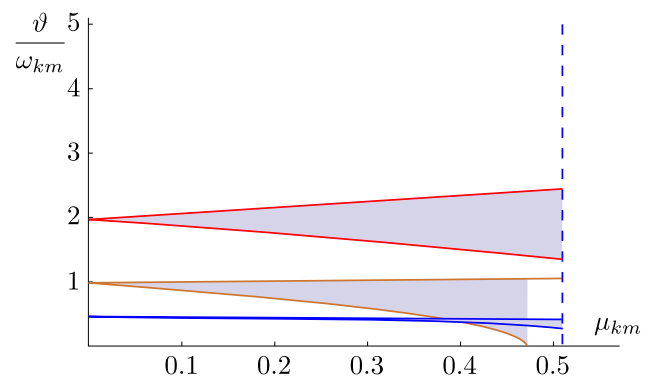

$\widetilde{\mu}_{k m}=0.51, n=0, \widetilde{\delta} / h=0, \theta_{0}=5$

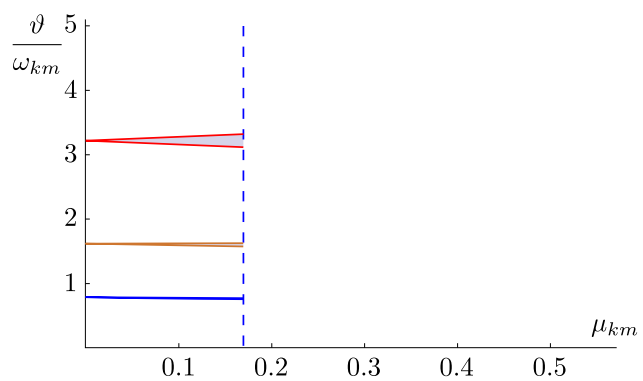

$\widetilde{\mu}_{k m}=0.17, n=3, h_{i} / h=5, \widetilde{\delta} / h=0, \theta_{0}=0$

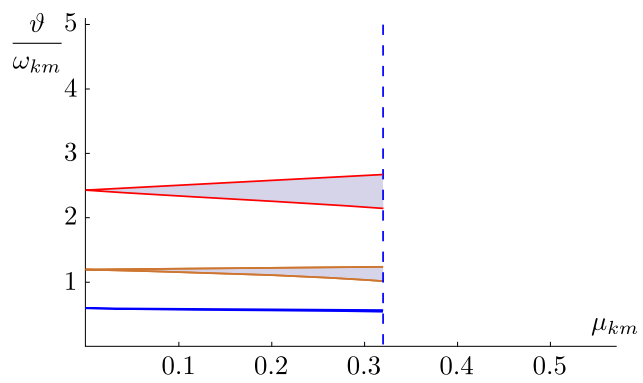

$\widetilde{\mu}_{k m}=0.32, n=1, h_{i} / h=5, \widetilde{\delta} / h=0, \theta_{0}=0$

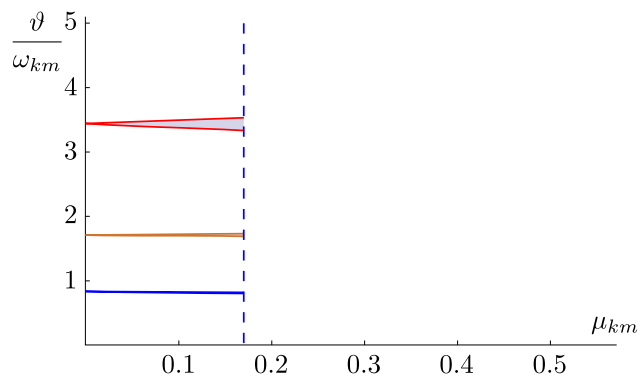

$\widetilde{\mu}_{k m}=0.17, n=0, \tilde{\delta} / h=2, \theta_{0}=0, \varepsilon_{1}=\varepsilon_{2}=1$

Конфигурация областей динамической устойчивости при заданных параметрах [Configuration of the dynamic stability domains depending on the given parameters] 

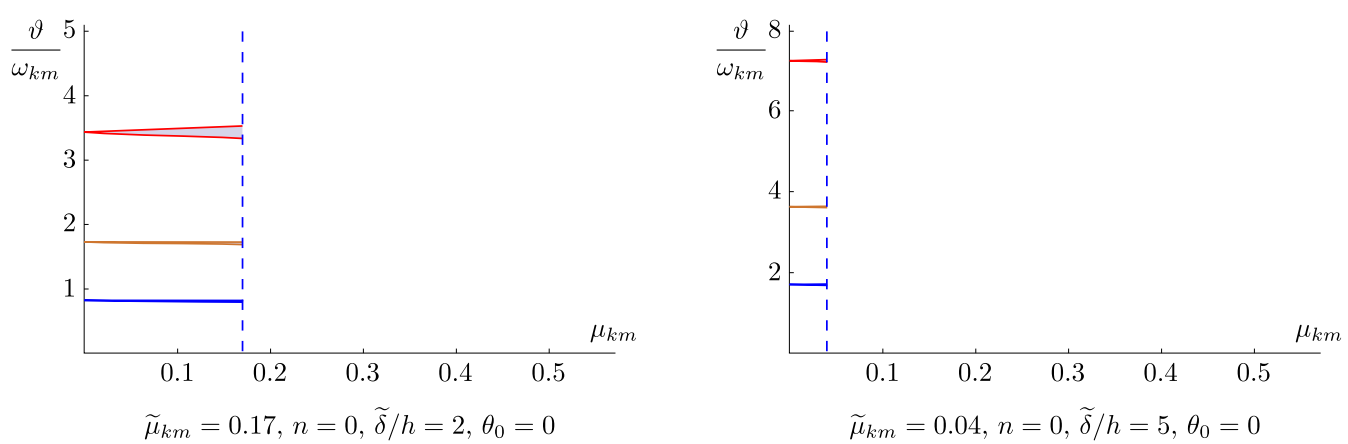

Конфигурация областей динамической устойчивости при заданных параметрах [Configuration of the dynamic stability domains depending on the given parameters]

Нетрудно убедиться, что тангенциальные усилия $T_{0}^{11}, T_{0}^{12}, T_{0}^{22}$ в этом случае не зависят от температуры $\theta_{0}$.

Анализ показывает, что конфигурации областей неустойчивости нечувствительны к подчеркнутым слагаемым в первых двух уравнениях системы (1). По этой причине удерживать их в уравнениях нет необходимости. Площади областей динамической неустойчивости существенно уменьшаются (как и предельные значения коэффициентов возбуждения) с увеличением параметров $h_{i} / h, n$ и $\widetilde{\delta} / h$ (при прочих равных условиях) при определенных значениях которых наблюдается вырождение областей неустойчивости в скелетные линии [16]. Следует также отметить возможность «замены» пологой гладкой цилиндрической оболочки на геометрически нерегулярную пластину (и обратно), подбирая соответствующим образом значения параметров $h_{i} / h$, $n$ и $\widetilde{\delta} / h$, что немаловажно для инженерной практики.

Конкурирующие интересы. Заявляем, что в отношении авторства и публикации этой статьи конфликта интересов не имеем.

Авторский вклад и ответственность. Все авторы принимали участие в разработке концепции статьи и в написании рукописи. Авторы несут ответственность за предоставление окончательной рукописи в печать. Окончательная версия рукописи была одобрена всеми авторами.

Финансирование. Результаты получены в рамках выполнения государственного задания Минобрнауки России № 9.8570.2017/8.9.

\section{Библиографический список}

1. Жилин П. А. Линейная теория ребристых оболочек // Изв. АН CCCP. MTT, 1970. № 4 . C. $150-162$.

2. Белосточный Г. Н., Ульянова О. И. Континуальная модель композиции из оболочек вращения с термочувствительной толщиной // Изв. РАН. МTT, 2011. № 2. С. 32-40.

3. Белосточный Г. Н., Русина Е. А. Оболочки и геометрически нерегулярные пластинки с термочувствительной толщиной // Докл. Росс. акад. естеств. наук, 1999. № 1. С. 28-37.

4. Абовский Н.П. О вариационных уравнения для гибких ребристых и других конструктивно-анизотропных пологих оболочек / Теория пластин и оболочек. М.: Наука, 1971. С. 4-7.

5. Назаров А. А. Основы теории и методы расчета пологих оболочек. Л., М.: Стройиздат, 1966. 
6. Antosik P., Mikusiński J., Sikorski R. Theory of Distributions: The Sequential Approach. Amsterdam: Elsevier Scientific, 1973.

7. Рассудов В. М. Деформации пологих оболочек, подкрепленных ребрами жесткости // Учен. зап. Сарат. ун-та, 1956. Т. 52. С. 51-91.

8. Геккелер И.В. Статика упругого тела. Л., М.: Гостехиздат, 1934.

9. Огибалов П. М. Вопросы динамики и устойчивости оболочек. М.: МГУ, 1963.

10. Огибалов П. М., Грибанов В. Ф. Термоустойчивость пластин и оболочек. М.: МГУ, 1958.

11. Белосточный Г.Н. Аналитические методы определения замкнутых интегралов сингулярных дифференциальных уравнений термоупругости геометрически нерегулярных оболочек // Доклады Академии военных наук, 1999. №1. С. 14-25.

12. Белосточный Г. Н., Русина Е. А. Динамическая термоустойчивость трансверсальноизотропных пластин под действием периодических нагрузок / Современные проблемы нелинейной механики конструкиий, взаимодействующих с агрессивными средами: Сб. науч. тр. межвуз. науч. конф.. Саратов, 2000. С. 175-180.

13. Белосточный Г. Н., Цветкова О. А. Геометрически нерегулярные пластинки под действием периодического по времени температурного поля/ Проблемы прочности элементов конструкций под действием нагрузок и рабочих сред. Саратов: Сарат. гос. техн. ун-т, 2002. 64-72 с.

14. Мыльцина О. А., Полиенко А. В., Белосточный Г. Н. Динамическая устойчивость нагретых геометрически нерегулярных пластин на основе модели Рейснера // Becmн. Сам. гос. техн. ун-та. Сер. Физ.-мат. науки, 2017. Т. 21, №4. С. 760-772. https:// doi.org/10.14498/vsgtu1579.

15. Stoker J. J. Nonlinear Vibrations in Mechanical and Electrical Systems/ Wiley Classics Library. New York: Wiley, 1992.

16. Болотин В. В. Динамическая устойчивость упругих систем. М.: ГИТТЛ, 1956.

17. Timoshenko S. P. Vibration Problems in Engineering. London: Constable, 1937.

18. Филиппов А. П. Методъ расчета сооружений на колебания. М., Л.: Госстройиздат, 1941.

19. Тимошенко С. П. Устойчивостъ упругих систем. М., Л.: ОГИЗ-Гостехизд, 1946.

20. Амбарцумян С. А. Теория анизотропных пластин. М.: Наука, 1967.

Математический институт им. В.А. Стеклова Российской академии наук приступает к работе в рамках Государственного контракта № 13.597.11.0043 по теме «Создание электронного архива выпусков научных журналов по тематическому направлению «Математика, физика, информационные технологии». Архив будет размещен на Общероссийском портале Math-Net.Ru.

Предполагается пополнить коллекцию Math-Net.Ru архивами ряда ведущих журналов по математике, физике и информационным технологиям, а также материалами научных мероприятий.

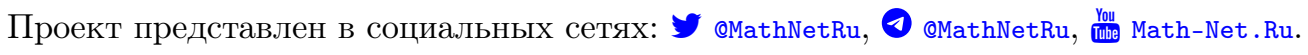




\title{
Dynamic thermal stability of heated geometrically irregular cylindrical shell under the influence of a periodic temporal coordinate load
}

\author{
(C) G. N. Belostochny, O. A. Myltcina
}

N. G. Chernyshevsky Saratov State University (National Research University), 83, Astrakhanskaya st., Saratov, 410012, Russian Federation.

\begin{abstract}
In the framework of a Love type model, a geometrically irregular isotropic shallow cylindrical shell is considered, based on a strict continuum-shell-rib model. It is assumed that the geometrically irregular shell is heated to a constant temperature $\theta_{0}$, two opposite edges are exposed to a tangential load periodic in time coordinate, the amplitude and frequency of which are known $\left(p(t)=p_{0} \cos \vartheta t\right)$. The problem of determining the regions of dynamic instability of a thermoelastic system is reduced to considering a singular system of three differential equations of dynamic thermal stability of a geometrically irregular shell in displacements containing a term with tangential forces in the Brian form. These forces arising in the shell during its heating are preliminarily determined on the basis of closed solutions of the singular system of differential equations of the momentless thermoelasticity of the geometrically irregular shell. The specific initialized system of equations is transformed to the Mathieu equations, which are written in terms of the classical athermal theory of smooth plates containing corrections for geometric parameters - curvature, relative height of the reinforcing elements, their number, and temperature. The first three regions of dynamic instability of a geometrically irregular shell are determined. A quantitative analysis of the influence of the geometric parameters of the elastic system and temperature on the configuration of the regions of dynamic instability and the magnitude of the excitation coefficient is carried out.
\end{abstract}

Keywords: singularity, thermal stability, dynamics, geometric irregularity, continuum model, Mathieu equations, closed integrals, instability domains.

Received: $14^{\text {th }}$ November, $2019 /$ Revised: $25^{\text {th }}$ June, $2020 /$

Accepted: $14^{\text {th }}$ September, $2020 /$ First online: $28^{\text {th }}$ September, 2020

\section{Short Communication}

(우(;) The content is published under the terms of the Creative Commons Attribution 4.0 International License (http://creativecommons.org/licenses/by/4.0/)

Please cite this article in press as:

Belostochny G. N., Myltcina O. A. Dynamic thermal stability of heated geometrically irregular cylindrical shell under the influence of a periodic temporal coordinate load, Vestn. Samar. Gos. Tekhn. Univ., Ser. Fiz.-Mat. Nauki [J. Samara State Tech. Univ., Ser. Phys. Math. Sci.], 2020, vol. 24, no. 3, pp. 583-594. https://doi.org/10.14498/vsgtu1755 (In Russian).

\section{Authors' Details:}

Grigory N. Belostochny (1) https://orcid.org/0000-0003-4471-6599

Dr. Techn. Sci.; Professor; Dept. of Mathematic Theory of Elasticity \& Biomechanics; e-mail: belostochny@mail.ru

Olga A. Myltcina (D) https://orcid.org/0000-0003-4718-2772

Cand. Phys. \& Math. Sci.; Associate Professor; Dept. of Functions \& Approxmation Theory; e-mail: omyltcina@yandex.ru 
Competing interests. We declare that we have no conflicts of interest in the authorship and publication of this article.

Authors' contributions and responsibilities. Each author has participated in the article concept development and in the manuscript writing. The authors are absolutely responsible for submitting the final manuscript in print. Each author has approved the final version of manuscript.

Funding. The results have been obtained within the State Assignment of the Ministry of Education and Science of the Russian Federation no. 9.8570.2017/8.9.

\section{References}

1. Zhilin P. A. The linear theory of ribbed shells, Izv. Akad. Nauk SSSR, Mekh. Tverd. Tela, 1970, no. 4, pp. 150-162 (In Russian).

2. Belostochnyi G. N., Ul'yanova O. I. Continuum model for a composition of shells of revolution with thermosensitive thickness, Mech. Solids, 2011, vol. 46, no. 2, pp. 184-191. https:// doi.org/10.3103/S0025654411020051.

3. Belostochnyi G. N., Rusina E. A. Shells and geometrically irregular plates with heat-sensitive thickness, Dokl. Ross. Akad. Estestv. Nauk, 1999, no. 1, pp. 28-37 (In Russian).

4. Abovskii N.P. On variational equations for flexible ribbed and other structurally anisotropic flat shells, Teoriia plastin i obolochek [Theory of Plates and Shells], 1971, pp. 4-7 (In Russian).

5. Nazarov A. A. Osnovy teorii i metody rascheta pologikh obolochek [Fundamentals of the Theory and Methods of Calculating Shallow Shells]. Leningrad, Moscow, Stroiizdat, 1966 (In Russian).

6. Antosik P., Mikusiński J., Sikorski R. Theory of Distributions: The Sequential Approach. Amsterdam, Elsevier Scientific, 1973.

7. Rassudov V. M. Deformations of gently sloping shells supported by stiffeners, Uchen. Zap. Sarat. Un-ta, 1956, vol. 52, pp. 51-91 (In Russian).

8. Gekkeler I.V. Statika uprugogo tela [Statics of an Elastic Body]. Leningrad, Moscow, Gostekhizdat, 1934 (In Russian).

9. Ogibalov P. M. Voprosy dinamiki i ustoichivosti obolochek [Problems in Dynamics and Stability of Shells]. Moscow, Moscow State Univ., 1963 (In Russian).

10. Ogibalov P. M., Gribanov V. F. Termoustoichivost' plastin i obolochek [Thermal Stability of Plates and Shells]. Moscow, Moscow State Univ., 1958 (In Russian).

11. Belostochny G. N. Analytical methods for determination of closed integrals of singular differential equations of thermoelasticity of geometrically irregular shells, Dokl. Akad. Voen. Nauk, 1999, no. 1, pp. 14-25 (In Russian).

12. Belostochny G. N., Rusina E. A. Dynamic thermal stability of transversal-isotropic plates under the action of periodic loads, In: Sovremennye problemy nelineinoi mekhaniki konstruktsii, vzaimodeistvuiushchikh s agressivnymi sredami [Modern problems of nonlinear mechanics of structures interacting with aggressive media]. Saratov, 2000, pp. 175-180 (In Russian).

13. Belostochny G. N., Tsvetkova O. A. Geometrically irregular plates under the action of a time-periodic temperature field, In: Problemy prochnosti elementov konstruktsii pod deistviem nagruzok $i$ rabochikh sred [Problems of strength of structural elements under the influence of loads and working media]. Saratov, Saratov State Techn. Univ., 2002, pp. 64-72 (In Russian).

14. Myltcina O. A., Polienko A. V., Belostochny G. N. Dynamic stability of heated geometrically irregular plates on the basis of the Reisner model, Vestn. Samar. Gos. Tekhn. Univ., Ser. Fiz.-Mat. Nauki [J. Samara State Tech. Univ., Ser. Phys. Math. Sci.], 2017, vol. 21, no.4, pp. 760-772 (In Russian). https://doi.org/10.14498/vsgtu1579.

15. Stoker J. J. Nonlinear Vibrations in Mechanical and Electrical Systems, Wiley Classics Library. New York, Wiley, 1992. 
16. Bolotin V. V. The dynamic stability of elastic systems, Holden-Day Series in Mathematical Physics. San Francisco, London, Amsterdam, Holden-Day, 1964.

17. Timoshenko S. P. Vibration Problems in Engineering. London, Constable, 1937.

18. Filippov A. P. Metody rascheta sooruzhenii na kolebaniia [Methods for Calculating Structures for Vibrations]. Moscow, Leningrad, Gosstroiizdat, 1941 (In Russian).

19. Timoshenko S. P. Ustoichivost' uprugikh sistem [Stability of Elastic Systems]. Moscow, Leningrad, OGIZ-Gostekhizd, 1946 (In Russian).

20. Ambartsumian S. A. Teoriia anizotropnykh plastin [Theory of Anisotropic Plates]. Moscow, Nauka, 1967 (In Russian). 